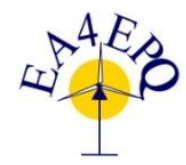

International Conference on Renewable Energies and Power Quality (ICREPQ'17)

Malaga (Spain), $4^{\text {th }}$ to $6^{\text {th }}$ April, 2017

Reneurable Energy and Pourer Quality. Fournal (RE\&PQJ)

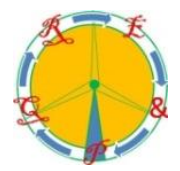

ISSN 2172-038 X, No.15 April 2017

\title{
On Sizing of Standalone Hybrid Wind/Solar/Battery Micro-grid System
}

\author{
U. Akram, M. Khalid and S. Shafiq \\ Department of Electrical Engineering \\ King Fahd University of Petroleum and Minerals (KFUPM) \\ Dhahran 31261, Kingdom of Saudi Arabia \\ e-mails: g201512930@kfupm.edu.sa, mkhalid@kfupm.edu.sa and saifullah@kfupm.edu.sa
}

\begin{abstract}
Capacity optimization of renewable energy sources such as solar and wind and the optimal sizing of associated battery energy storage (BES) is essential for economic and reliable operation of a standalone micro-grid (MG). In this work a constraint based iterative search algorithm is proposed to find the optimal sizes of solar, wind and battery capacity in a standalone MG. The proposed method is based on two basic principles, i.e., maximum reliability and minimum cost. The proposed technique is robust and guarantees to avoid the over and under sizing. In addition it considers the forced outage rates (FORs) of solar and wind and utilization factor of battery storage which makes it more practical. Renewables and demand data of Dammam region of KSA is used to test the proposed method. A reliability and economic analysis is also carried out to validate the proposed technique.
\end{abstract}

\section{Key words}

Micro-grid, wind power, solar power, battery energy storage, optimization

\section{Introduction}

Most recently, utilizing fossil fuels such as natural gas, oil and coal produces major proportion of electricity. Burning of fossil fuels adds massive amount of carbon in the atmosphere. Moreover, fossil fuels reserves are depleting rapidly and their prices are fluctuating. On the other hand, demand is increasing and new environmental policies are also being made to mitigate carbon emission problems. So a decrease in the use of fossil fuels and search for other alternatives are essential for reliable and eco-friendly power generation. Renewable energy sources (RES) can be used as a substitute of fossil fuels as they provide green energy and are freely available. Remote areas such as Islands, which are far away from the utility grid, can also utilize local available green energy sources to meet their demand [1], [2].

Wind and solar are most widely used renewable sources for the generation of electricity [3]. Output of wind turbine (WT) and solar photovoltaic (PV) are highly variable because they harvest energy from natural resources which have low controllability [4], [5]. The intermittent output and reliability problems associated with RES can potentially be addressed by using energy storage systems (ESS) [6]-[9]. In particular, battery energy storage (BES) can be employed for energy storage in a system utilizing renewables [10]-[12].

A hybrid wind solar generation system utilizes both wind and solar to extract the maximum available energy. It is more reliable and efficient than solar or wind alone. In addition a hybrid wind-solar generation system requires less storage capacity making it more economical [13]. In addition to these benefits, the most important is to design a micro-grid (MG) system with optimal sizing of WT, PV and BES for efficient, reliable and economical operation.

Several methods have been developed for optimal sizing of WT, PV and BES. In [14], an iterative technique is proposed based on minimizing the difference between the generation and load. This proposed technique may lead to oversizing of PV, WT and BES. In [15], optimal size is determined considering multi-objectives, i.e., high supply reliability and minimization of cost. The major problem in this method is that it sets the minimum limit for PV and WT to supply the average demand during day and night times respectively. This assumption may lead to incorrect optimal solution. Moreover complementary characteristics of wind and solar are considered which is not the case for all the geographical locations is. This limits the proposed method to the areas, which have complementary characteristics. In [16], optimal sizing is done considering the reliability and cost objectives. Particle swarm optimization (PSO) is used to find the optimal sizes of grid connected PV, WT and BES considering reliability cost and emissions objectives [17].

In this paper, an innovative optimization methodology based on advanced constraint-based iterative search algorithm is proposed for the capacity optimization of solar PV, WT, and BES in a standalone MG system. The proposed algorithm generates a search space consisting of different combinations of PV, WT and BES. The optimal solution is then obtained from the given search space on 
the basis of reliability and cost ratio. The proposed method is robust as so there is no over-sizing and undersizing problem. Moreover the technique is general and can be applied to other types of generations and storages.

The remainder of the paper is organized as follows. Section 2 discusses about the structure and mathematical model of standalone MG. Section 3 describes the proposed methodology. Section 4 presents the results while conclusion is given in Section 5.

\section{Micro-grid}

A MG is a small scale power station that utilizes locally available resources to fulfil the required load which can be operated along with main grid or in standalone mode. In this study standalone MG is considered which is shown in Fig. 1. PVs and WTs are employed for hybrid power generation while for storage battery technology is utilized. $\mathrm{PV}$ and WT and BES are connected to DC bus via DC/DC and $\mathrm{AC} / \mathrm{DC}$ and $\mathrm{DC} / \mathrm{DC}$ converters respectively. The DC bus is connected to load bus through DC/AC converter and a transformer.

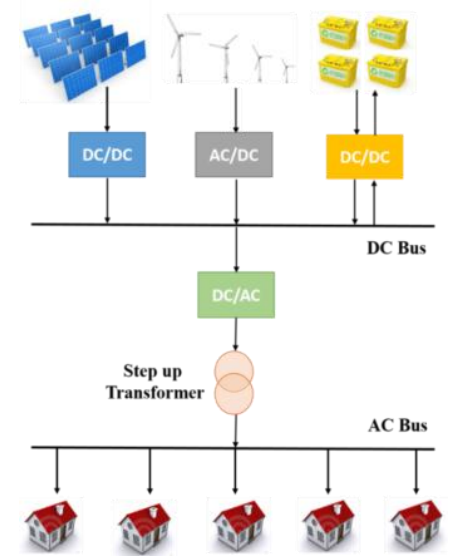

Fig. 1. A typical stand-alone MG system.

\section{A. Wind System}

A WT transforms the kinetic energy of moving wind to electrical energy. The power output of a WT can be calculated using the following equation [18].

$P_{W T}(v)=\left\{\begin{array}{l}0 \\ P_{\text {rated }}\end{array} \times \frac{v-v_{c i}}{v_{r}-v_{c i}}\right.$

$$
\begin{gathered}
v<v_{c i} \\
v_{c i} \leq v<v_{r} \\
v_{r} \leq v<v_{c o} \\
v \geq v_{c o}
\end{gathered}
$$

where $v_{r}, v_{c i}$, and $v_{c o}$ are rated, cut-in and cut-out wind speeds respectively. The WT generates no power below the cut-in speed, and between cut-in and cut-out speeds power output increases with the speed. The WT generates $P_{\text {rated }}$ between rated and cut-out speeds, and no power beyond cut-out speed.

\section{B. Solar PV System}

A solar PV system converts solar energy to electrical energy. The power output of PV system depends on solar irradiation, area of the PV array, atmospheric temperature, and its efficiency. In this study a linear relation between the solar irradiation and output power of PV is considered.

\section{Battery Energy Storage System}

The BES system is composed of series and parallel connected battery banks. The normalized energy of BES can be calculated as

$$
\begin{gathered}
E_{B}(t)=E_{B}(t-1)+\eta_{B} \times \sum\left\{E_{G}(t)-E_{L}(t)\right\} \\
(1-D O D) \leq E_{B}(t) \leq 1
\end{gathered}
$$

where $\eta_{B}$ is the charging/discharging efficiency of BES, $D O D$ is the depth of discharge, and $E_{G}, E_{B}$ and $E_{L}$ are energy supplied generating sources, energy supplied by BES and energy demand respectively.

\section{Proposed Methodology}

The power generated by a MG system utilizing PVs and WTs can calculated as:

$$
\begin{gathered}
P_{G}^{(i, j)}(t)= \\
N_{P V}^{i} P_{P V}(t) P V_{\text {status }}^{i}(t)+ \\
N_{W T}^{j} P_{W T}(t) W T_{\text {status }}^{j}(t) \\
N_{P V}^{\min } \leq N_{P V}^{i} \leq N_{P V}^{\max } \\
N_{W T}^{\min } \leq N_{W T}^{j} \leq N_{W T}^{\max }
\end{gathered}
$$

where $N_{W T}$ and $N_{P V}$ are number of WTs and PVs respectively, $P_{W T}$ is the output power of one WT and $P_{P V}$ is the output power of one PV, $W T_{\text {status }}$ and $P V_{\text {status }}$ are the status of WT and PV which are calculated based on FORs and $N_{W T}^{\min }, N_{P V}^{\min }, N_{W T}^{\max }$ and $N_{P V}^{\max }$ are minimum and maximum number of WTs and PVs. The instantaneous error $\Delta p(t)$ is the difference between the generation and demand at any instant of time can be calculated using the following equation

$$
\Delta p^{(i, j)}(t)=P_{L}(t)-P_{G}^{(i, j)}(t)
$$

where $P_{L}$ is the system demand. The total error $\Delta P$ can be calculated by summing the absolute values $\Delta p$. The cumulative error matrix $\Delta \boldsymbol{P}$ contains $\Delta P$ for all possible combinations of $N_{W T}$ and $N_{P V}$ and $\boldsymbol{N}_{\boldsymbol{W T}}$ and $\boldsymbol{N}_{\boldsymbol{P V}}$ are $(i \times 1)$ and $(1 \times j)$ vectors that contain all values number of WTs and PVs that are considered in this work. A search space can be formed using $\Delta \boldsymbol{P}$ and $\boldsymbol{N}_{\boldsymbol{P V}}$ and $\boldsymbol{N}_{\boldsymbol{W T}}$. This search space can be reduced by applying the constraint, $\Delta \boldsymbol{P} \stackrel{\min }{\Longrightarrow} \Delta \boldsymbol{P}_{\min }$. The reduced search space is given as

$$
R S_{\text {space }}=\left[\begin{array}{lll}
\Delta \boldsymbol{P}_{\min } & \boldsymbol{N}_{\boldsymbol{P V}}^{\min } & \boldsymbol{N}_{W T}^{\min }{ }^{T}
\end{array}\right]_{j \times 1}
$$

where $R S_{\text {space }}$ is the reduced search space, $\Delta \boldsymbol{P}_{\min }$, is a $(1 \times j)$ vector that contain the cumulative errors that satisfy the constraint and $\boldsymbol{N}_{W T}^{m i n}$ and $\boldsymbol{N}_{P V}^{m i n}$ contain the corresponding values of $N_{W T}$ and $N_{P V}$. The power 
generated by case $u$ chosen from $R S_{\text {space }}$ is $P_{G}^{u}(t)$ which can be calculated as

$$
P_{G}^{u}(t)=N_{P V}^{\min ^{u}} P_{P V}(t)+N_{W T}^{\min ^{u}} P_{W T}(t)
$$

The gap in load and generation at any instant of time is given as

$$
p_{\text {gap }}^{u}(t)=P_{L}(t)-P_{G}^{u}(t)
$$

The $P_{g a p}^{u}$ is a vector whose entries are non-negative numbers and each entry is the summation of all the previous entries. It is calculated using (8)

$$
\begin{gathered}
P_{\text {gap }}^{u}(r)=\sum_{t=1}^{r} p_{\text {gap }}^{u}(t) \\
P_{\text {gap }}^{u}(r)= \begin{cases}P_{\text {gap }}^{u}(r) & P_{\text {gap }}^{u}(r)>0 \\
0 & \text { else }\end{cases}
\end{gathered}
$$

The maximum BES capacity that can be installed to store the maximum of surplus energy can be calculated by using the following expression

$$
B_{\text {max }}^{u}=\max \left(P_{\text {gap }}^{u}\right)
$$

where $B_{\max }^{u}$ is the maximum BES capacity for the case $u$. The required BES capacity is less than or equal to $B_{\max }^{u}$ is calculated as

$$
B_{\text {cap }}^{u}= \begin{cases}B_{\text {max }}^{u} & x=1 \\ C B S^{u} & x=0\end{cases}
$$

where $B_{c a p}^{u}$ is the required BES capacity and it is equal to $B_{\max }^{u}$ if the battery discharges fully to its minimum allowable point after its complete charging, this condition is indicated by $x=1$. Otherwise $B_{\text {cap }}^{u}$ will be equal to corrected battery size $(C B S)$. The $C B S$ is determined using the region reduction algorithm. Finally the optimal capacity of BES is determined based on the utilization factor.

$$
B_{\text {optimal }}^{u}=\left\{\begin{array}{lc}
B_{\text {cap }}^{u} & U F^{u} \geq B U_{\text {limit }} \\
B C S^{u} & \text { else }
\end{array}\right.
$$

where $B_{\text {optimal }}^{u}$ is the optimal BES size. If $U F^{u}$ is less than $B U_{\text {limit }}$, $B_{\text {optimal }}^{u}$ will be equal to battery corrected size $\left(B C S^{u}\right)$. The $B C S$ is calculated using an iterative algorithm.

Reliability and cost can be used to assess the performance of any system. In this study a ratio of energy served and cost is used to find the optimal solution. The total cost $C_{t}$ can be determined as

$$
C_{t}^{u}=C_{c}^{u}+C_{m}^{u}
$$

where $C_{m}$ and $C_{c}$ are maintenance and capital costs respectively. The cost of generation can be calculated by using the following equation

$$
C_{g}^{u}=\frac{\sum_{i=1}^{N} N P V_{i}^{u}}{\sum_{i=1}^{N} N D E S_{i}^{u}}
$$

where $C_{g}$ and $\mathrm{N}$ is the generation cost and the total number of renewable sources respectively. The optimal decision variable $(O D V)$ can be calculated as

$$
O D V^{u}=\frac{E_{s}^{u}}{C_{u n i t}^{u}}
$$

where $C_{\text {unit }}$ and $E_{s}$ are cost per unit and the energy served respectively. The solution vector $(S V)$ is a row vector given as

$$
S V=\left[\begin{array}{llll}
O D V^{1} & \cdots & O D V^{j-1} & O D V^{j}
\end{array}\right]
$$

The optimal WT, PV and BES combination corresponds to the index of maximum value of $S V$.

\section{Results and Discussions}

The proposed methodology is tested using residential demand and renewables data of Dammam city which is situated in the Eastern province of Kingdom of Saudi Arabia. Time series data of both renewables and demand for a length one year and with a resolution of one hour is used. The demand data is normalized to a peak of 10MW. The normalized average daily demand is shown in Fig. 2. The minimum daily load demand appears around 6 am to 8 am which increases after that and daily peak demand occurs at around $8 \mathrm{pm}$. The normalized daily average wind power and solar are shown in Fig. 3 and Fig. 4 respectively. It can be observed from that solar gives power during the day time while wind power keeps on fluctuating throughout the day.

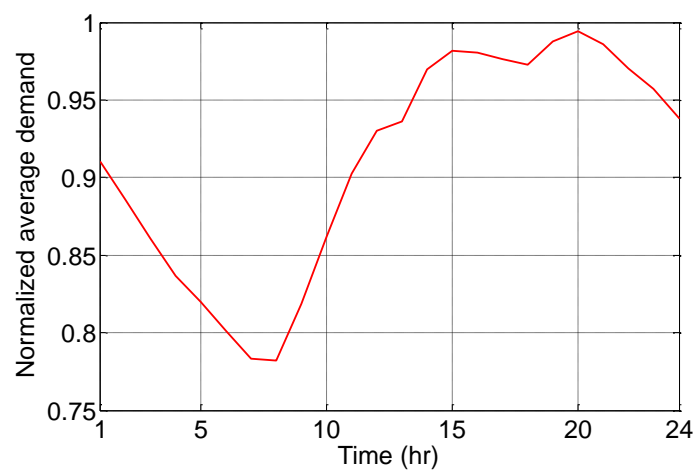

Fig. 2. Normalized daily average demand.

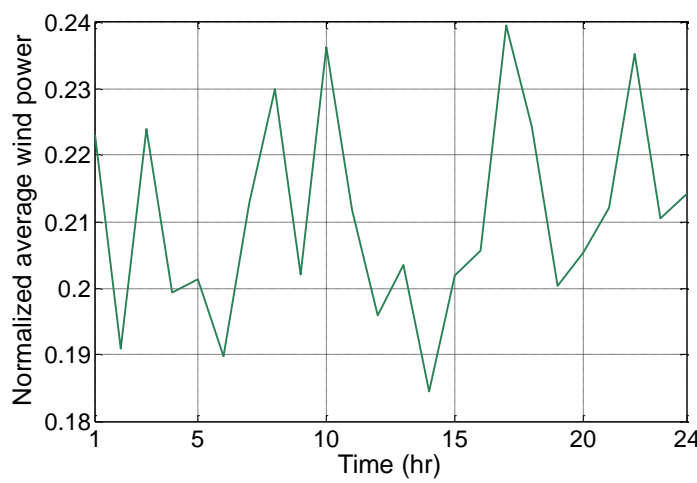

Fig. 3. Normalized average wind power profile. 


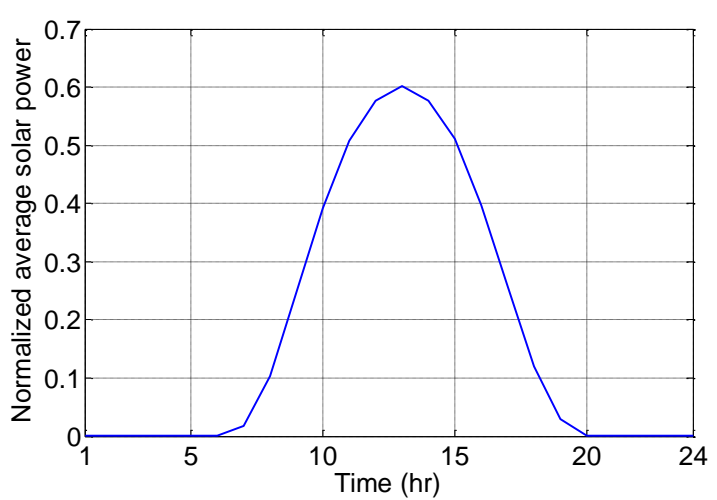

Fig. 4. Normalized average solar PV power profile.

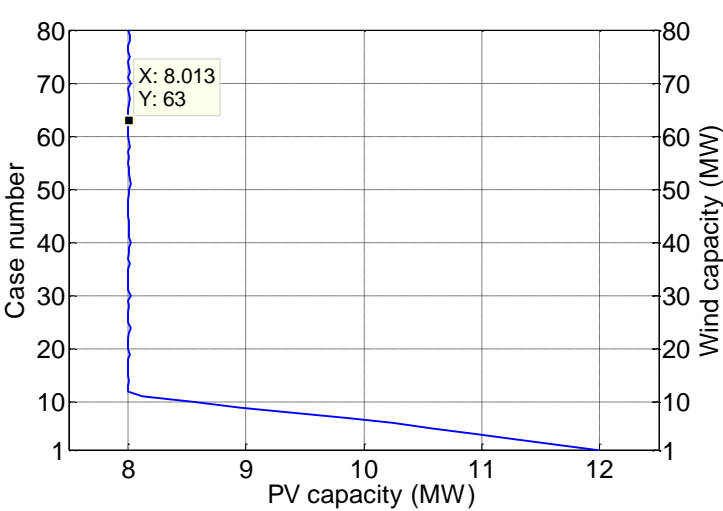

Fig. 5. capacities combinations wind and solar.

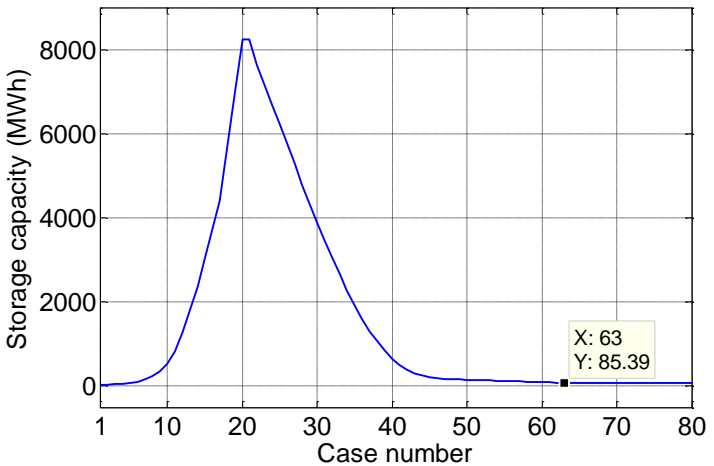

Fig. 6. Optimal storage size.

In this study the $R S_{\text {space }}$ consists of 80 cases. The capacities for WT and PV corresponding to each case are shown in Fig. 5. The optimal BES capacity for each combination of WT and PV is presented in Fig. 6. It is clear from Fig. 5 and Fig. 6 that the cases for which overall installed capacity is small and very large have small size of energy storage.

The performance of any system can be analyzed using its cost and reliability. In this study an economic and reliability analysis of the cases selected for the $R S_{\text {space }}$ is carried out in order to find the optimal solution. The optimal solution is selected on the basis of energy served to cost ratio. A high value of ratio means higher reliability at relatively lower cost. The case which has the maximum ratio will be the optimal solution provided that unserved energy must be approximately zero. The energy served to cost ratio is shown in Fig. 7 and energy served and energy unserved are shown in Fig. 8. It can be observed from the Fig. 7 and Fig. 8 that the local maximum appears for the case 3 but it cannot be selected as an optimal solution because the corresponding energy unserved is very large. The global maximum appears for the case number 63 and the unserved energy for this case is almost zero which is depicted in the Fig. 7 and Fig. 8. The demanded energy for the whole year is successfully served for the optimal case. The WT, PV and BES capacities corresponding to the optimal case can be obtained from Fig. 6 and Fig. 7.

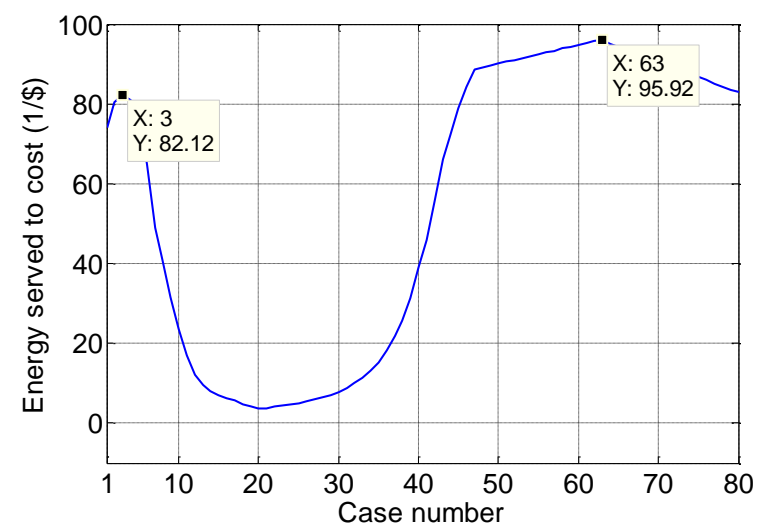

Fig. 7. Energy served to cost ratio.

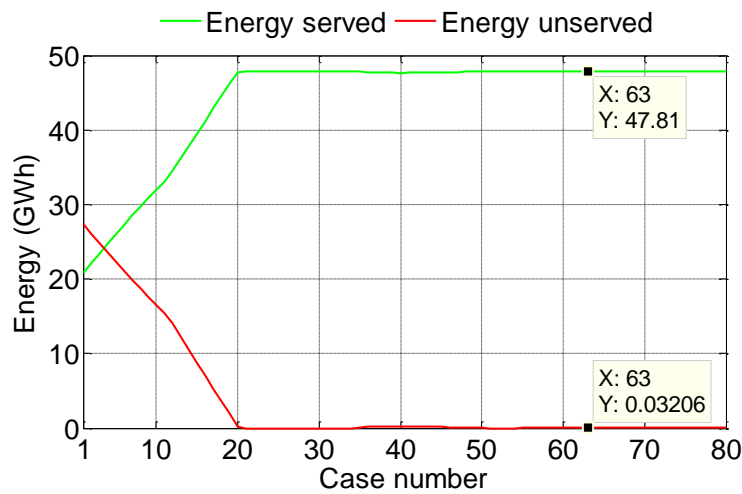

Fig.8. Energy served and energy unserved comparison.

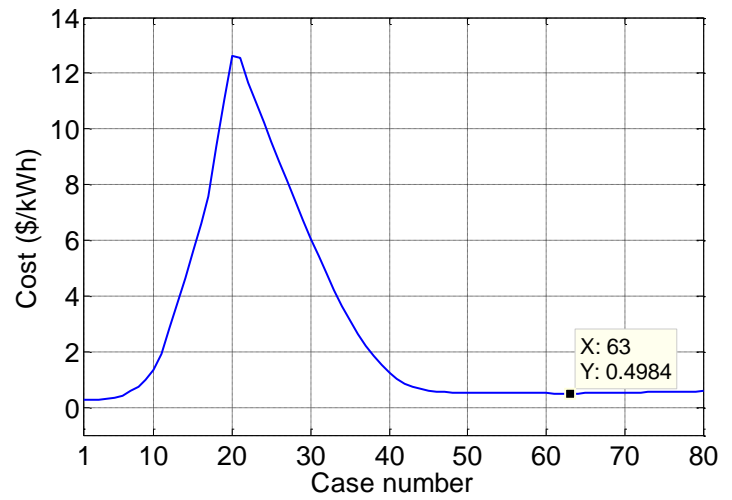

Fig.9. System estimated Cost per unit.

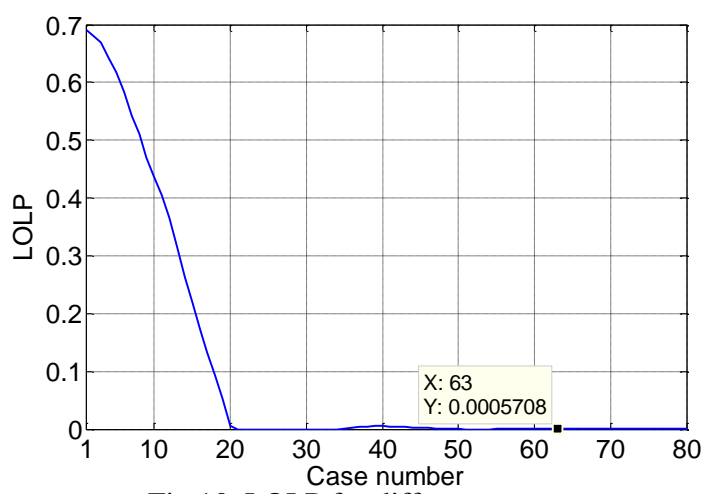

Fig.10. LOLP for different cases. 
The cost per unit and LOLP for different cases are shown in Fig. 9 and Fig. 10. It is evident from these figures that the cost is reasonable and LOLP is almost zero for the optimal solution. It can be concluded from the Fig. 6 and Fig. 10 that cost is highly dependent upon the storage size. The utilization factor of BES for the cases is shown in Fig. 11. It can be seen that utilization factor is greater than $80 \%$ for all the cases as per constraints.

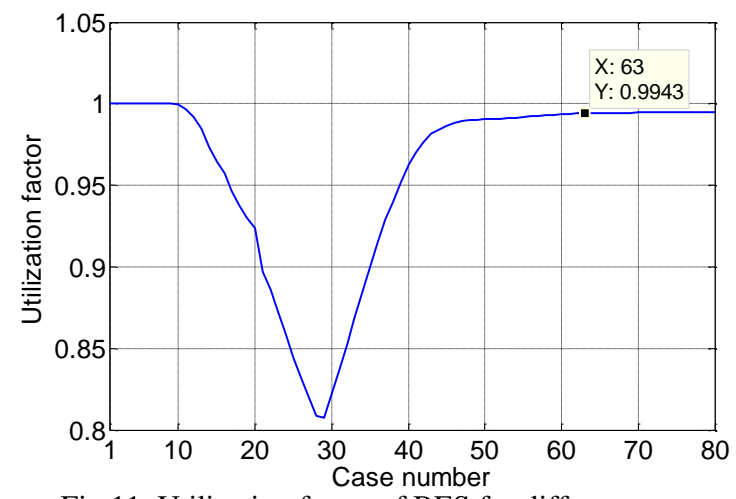

Fig.11. Utilization factor of BES for different cases.

\section{Conclusion}

In this paper, a methodology is proposed for the optimal sizing of solar, wind and BES in a stand-alone MG system. The proposed technique avoids under and over sizing as it searches every possible solution. Moreover, to make the technique more realistic, FORs of PV and WT and utilization factor of BES is also considered.

The proposed technique is validated using solar, wind and load data of Dammam. Reliability and cost analysis are performed and compared for different cases. The optimal solution is determined on the basis of reliability to cost ratio. LOLP and energy unserved are almost zero and the cost per unit is reasonable for optimal solution.

\section{Acknowledgement}

We thank the support provided by Research Institute at King Fahd University of Petroleum and Minerals and Saudi Electricity Company for providing time series data.

\section{References}

[1] C. Li, X. Liu, Y. Cao, P. Zhang, H. Shi, L. Ren, and Y. Kuang, "A time-scale adaptive dispatch method for renewable energy power supply systems on Islands," IEEE Transactions on Smart Grid, vol. 7, no. 2, pp. 1069-1078, 2016.

[2] T. Ma, H. Yang, and L. Lu, "A feasibility study of a standalone hybrid solar-wind-battery system for a remote Island," Applied Energy, vol. 121, pp. 149-158, 2014.

[3] Y. Zhu, B. Liu, and X. Sun, "Frequency-based power management for PV/battery/fuel cell stand-alone microgrid," in 2nd InternationalFuture Energy Electronics Conference (IFEEC). IEEE, 2015, pp. 1-6.

[4] B. Lynch, A. Kiger, and K. Hatipoglu, "An overview of hydrogen electrical energy storage meeting energy demand with intermittent renewable resources," in IEEE SoutheastConf, 2016. IEEE, 2016, pp. 1-6.

[5] B. Zhou and T. Littler, "Local storage meets local demand: a technical solution to future power distribution system,"
IET Generation, Transmission \& Distribution, vol. 10, no. 3, pp. 704-711, 2016.

[6] P. Denholm and R. M. Margolis, "Evaluating the limits of solar photovoltaics (pv) in electric power systems utilizing energy storage and other enabling technologies," Energy Policy, vol. 35, no. 9, pp. 4424-4433, 2007.

[7] B. Yang, Y. Makarov, J. Desteese, V. Viswanathan, P. Nyeng, B. McManus, and J. Pease, "On the use of energy storage technologies for regulation services in electric power systems with significant penetration of wind energy," in 5th International Conference on the European Electricity Market. IEEE, 2008, pp. 1-6.

[8] A. Khatamianfar, M. Khalid, A. V. Savkin, and V. G. Agelidis, "Improving wind farm dispatch in the Australian electricity market with battery energy storage using model predictive control," IEEE Transactions on Sustainable Energy, vol. 4, no. 3, pp.745-755, 2013.

[9] M. Khalid and A. V. Savkin, "A model predictive control approach to the problem of wind power smoothing with controlled battery storage," Renewable Energy, vol. 35, no. 7, pp. 1520-1526, 2010.

[10] S. Shafiq and A. T. Al-Awami, "Reliability and economic assessment of renewable micro-grid with V2G electric vehicles coordination," in Jordan Conference on Applied Electrical Engineering and Computing Technologies (AEECT). IEEE, 2015, pp. 1-6.

[11] A. V. Savkin, M. Khalid, V. G. Agelidis, "A Constrained monotonic charging/discharging strategy for optimal capacity of battery energy storage supporting wind farms," IEEE Transactions on Sustainable Energy, vol. 7, no. 3, pp.1224-1231, 2016.

[12] M. Khalid, A. Ahmadi, A. V. Savkin, and V. G. Agelidis, "Minimizing the energy cost for microgrids integrated with renewable energy resources and conventional generation using controlled battery energy storage', Renewable Energy, vol. 97, pp. 646-655, 2016.

[13] Z. Benhachani, B. Azoui, R. Abdessemed, and M. Chabane, "Optimal sizing of a solar-wind hybrid system supplying a farm in a semi-arid region of algeria," in 47th International Universities Power Engineering Conference (UPEC). IEEE, 2012, pp. 1-6.

[14] W. Kellogg, M. Nehrir, G. Venkataramanan, and V. Gerez, "Generation unit sizing and cost analysis for stand-alone wind, photovoltaic, and hybrid wind/PV systems," IEEE Transactions on Energy Conversion, vol. 13, no. 1, pp. 7075, 1998.

[15] L. Xu, X. Ruan, C. Mao, B. Zhang, and Y. Luo, “An improved optimal sizing method for wind-solar-battery hybrid power system," IEEE Transactions on Sustainable Energy, vol. 4, no. 3, pp. 774-785, 2013.

[16] H. Yang, L. Lu, and W. Zhou, "A novel optimization sizing model for hybrid solar-wind power generation system," Solar Energy, vol. 81, no. 1, pp. 76-84, 2007.

[17] L. Wang and C. Singh, "Pso-based multi-criteria optimum design of a grid-connected hybrid power system with multiple renewable sources of energy," in IEEE Swarm Intelligence Symposium. IEEE, 2007, pp. 250-257.

[18] S. Roy, "Market constrained optimal planning for wind energy conversion systems over multiple installation site," IEEE Transactions on Energy Conversion, vol. 17, no. 1, pp. 124-129, 2002. 